\title{
OPEN Molecular dynamics study on the relationship between phase transition mechanism and loading direction of AZ31
}

\author{
Qianhua Yang ${ }^{1}$, Chun Xue ${ }^{1}$, Zhibing Chu ${ }^{1 凶}$, Yugui Li ${ }^{1}$, Lifeng $\mathrm{Ma}^{2}$ \& Hong Gao ${ }^{3}$
}

To develop and design mg-based nanoalloys with excellent properties, it is necessary to explore the forming process. In this paper, to explore the effect of different loading directions on the phase transformation of magnesium alloy, the model of AZ31 magnesium alloy was established, the process of Uniaxial Compression (UC) of magnesium alloy in different directions was simulated, the changes of atomic position and phase structure were observed, and the phase transformation mechanism of AZ31 magnesium alloy under uniaxial compression under different loading directions was summarized. The conclusions are as follows: the stress and strain, potential energy and volume change, void evolution, phase structure change and dislocation evolution of magnesium alloy are consistent, and there is no significant difference. In the process of uniaxial compression, the phase transformation of hexagonal closely packed (HCP) $\rightarrow$ face-centered cubic $(F C C)$ is the main, and its structure evolves into $\mathrm{HCP} \rightarrow$ Other $\rightarrow$ FCC. Shockley partial dislocations always precede FCC stacking faults by about $4.5 \%$, and Shockley partial dislocations surround FCC stacking faults. In this paper, the phase transformation mechanism of AZ31 magnesium alloy under uniaxial compression under different loading directions is summarized, which provides a theoretical basis for the processing and development of magnesiumbased nanoalloys.

Magnesium alloys are commonly used in the aerospace and automotive industry because of their low density and excellent mechanical properties ${ }^{1-8}$. However, magnesium alloy shows obvious anisotropy during deformation at room temperature, because its hexagonal closely packed (HCP) structure has less slip system ${ }^{9}$. Nanocrystalline metals and alloys will show different characteristic mechanical properties from coarse-grained metals and alloys. Compared with coarse-grained polycrystalline materials, nanocrystalline materials show higher strength, hardness, and toughness. Nanostructure will be an effective way to improve the strength and plasticity of magnesium alloys in the future ${ }^{10-16}$. Therefore, it is more and more necessary to study the changes of microstructure and mechanical properties in the process of material processing from the basic process of molecular motion ${ }^{17}$. With the progress of science and technology, computer simulation is widely used to explore the microstructure and mechanical properties of materials, and the typical application is molecular dynamics simulation (MD) ${ }^{18-23}$. as a new means of calculating materials, molecular dynamics simulation has been successfully used to study lattice distortion, grain deformation, and so on ${ }^{24}$.

Sunil Rawat ${ }^{25}$ studied the plasticity (twin and dislocation slip) and $\omega$ phase transition of single crystal titanium under different strain directions by molecular dynamics simulation. The loading perpendicular to the $c$ axis leads to the activation and $\omega$ phase transition of $\{10 \overline{12}\}$ twins. For loading in the [2/10] direction, four twin variables (two conjugate pairs) are activated, and for loading in the [01 $\overline{1} 0]$ direction, two twin variables (one conjugate pair) are activated. Xiaoqin $\mathrm{Ou}^{26}$ uniaxial tensile tests of nano-face-centered cubic iron were carried out along [112], [1 $\overline{1} 0]$ and [111], respectively, through molecular dynamics simulation. The applied tension along with the [112] direction leads to the transformation of the body-centered cube in the face-centered vertical direction. The stable cubic core of flat ellipsoid is composed of $(01 \overline{1})$ body-centered cubic twin martensite structure. The stretching direction is [1 $\overline{1} 0]$, and the two groups of plate-centered cubic fibers perpendicular to each other form a face-centered cubic phase with the matrix. The tensile direction is [111], and a large number of stacking faults appear temporarily due to the movement of partial dislocations in Shockley. The stacking fault is funnel-shaped

${ }^{1}$ College of Materials Science and Engineering, Taiyuan University of Science and Technology, Taiyuan 030024, China. ${ }^{2}$ College of Mechanical Engineering, Taiyuan University of Science and Technology, Taiyuan 030024, China. ${ }^{3}$ Jiangsu Wujin Stainless Steel Co., Ltd, Changzhou 213000, China. ${ }^{\varpi}$ email: s20190347@stu.tyust.edu.cn 


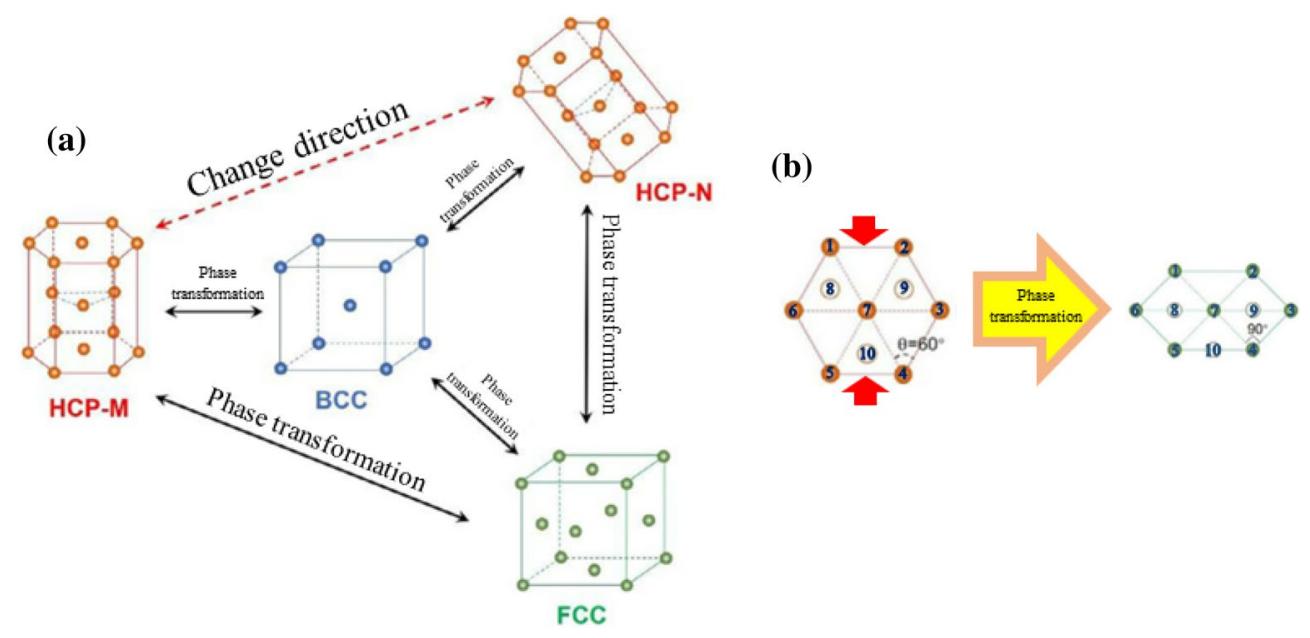

Figure 1. Phase transformation principle of AZ31 magnesium alloy.

and consists of two "Pyramids" in relative positions. Satyajit Mojumder ${ }^{27}$ has studied the compression load effect of different $\mathrm{Al}$ crystal orientations on the plasticity of $\mathrm{Al}-\mathrm{Cu}$ alloy by molecular dynamics method. For $\langle 001\rangle$ direction, the alloy can bear higher stress, while $\langle 110\rangle$ direction shows the opposite trend. For $\langle 111\rangle$ direction, the medium alloying degree has the highest strength. The elastic modulus in $\langle 111\rangle$ direction is the highest and the yield strain is the smallest, while the yield stress in $\langle 110\rangle$ direction is the highest and the yield strain is the largest. Hao Zhang ${ }^{28}$ has studied the effect of uniaxial tensile direction on the deformation mechanism of hexagonal closely packed (HCP) titanium crystals by molecular dynamics simulation. It is found that a series of "HCP $\rightarrow$ BCC $\rightarrow$ FCC" phase transitions occur when the tensile load is in the direction of [ $\overline{2} 110]$; when the

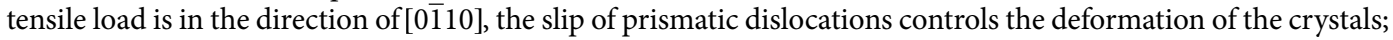
the prismatic dislocations can be dissociated into Shockley dislocations on the base, resulting in the formation of basal stacking faults. When the tensile load is in the direction of [0001], a large number of $\langle c+a\rangle$ incomplete dislocations and Shockley partial dislocations are formed in the crystal, which is derived from the dissociation of $\langle c+a\rangle$ dislocations.

Figure 1a shows the possible phase transition of AZ31 magnesium alloy during compression. When the magnesium alloy is compressed, the HCP structure of magnesium alloy changes and may turn from the original HCP-M phase to an angled HCP-N phase, or caused by the change of atomic position, one is to transform into BCC phase, the other is to transform into FCC phase. Similarly, the BCC phase or FCC phase can also form the $\mathrm{HCP}$ phase due to the change of atomic position. Figure $1 \mathrm{~b}$ is the phase transition mechanism of $\mathrm{HCP} \rightarrow$ FCC. It is found that when the HCP structure is compressed, the distance of atoms in the horizontal direction increases, and the distance in other directions shortens. The positions of atoms 8,9 , and 10, which were originally located in the interior of the HCP structure, do not change. However, due to the shortening of the distance between atoms 1 and 5, 2 and 4, atoms 8, 9, and 10 are already on the surface. The $\angle 347$ originally is $60^{\circ}$, but now is $90^{\circ}$. At this time, the HCP structure becomes the FCC structure, and the FCC phase transition occurs.

To sum up, there is a lack of research on the relationship between phase transformation and loading direction of magnesium alloy. Therefore, to explore the effect of different loading directions on the phase transformation of magnesium alloy, the model of AZ31 magnesium alloy is established, the process of uniaxial compression (Uniaxial Compression, UC) of magnesium alloy along [ $\overline{1} 2 \overline{1} 0],[\overline{1} 010]$ and [0001] directions are simulated, the changes of atomic position and phase structure are observed. The phase transformation mechanism of AZ31 magnesium alloy under uniaxial compression under different loading directions is summarized. and the phase transformation mechanism of AZ31 magnesium alloy under different loading directions is summarized. It provides a theoretical basis for the development of new magnesium-based nano-alloys and an idea for new processing methods of magnesium alloys.

\section{Simulation means and methods}

LAMMPS refers to a large-scale atomic/molecular massively parallel simulator, which can simulate the processing of nanomaterials ${ }^{29}$. The potential function is used to describe the relationship between the total energy $E$ of the model particles and the $(r)$ coordinates of the particles ${ }^{30-32}$. The embedded atomic potential (EAM) is usually used to calculate the interaction between metal and metal alloy atoms. The improved embedded atomic potential (MEAM) can be used to calculate the pairwise interactions of metals and alloys with FCC, BCC, HCP, and Diamond structures ${ }^{33-39}$. The total energy $E$ of the atomic system is shown in the formula:

$$
E=\sum_{i}\left\{F_{i}\left(\bar{\rho}_{i}\right)+\frac{1}{2} \sum_{i \neq j} \phi_{i j}\left(\gamma_{i j}\right)\right\} .
$$




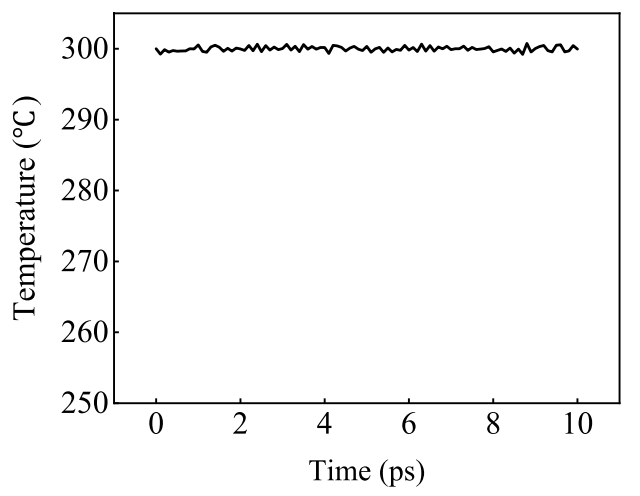

Figure 2. The MSD curve of relaxation temperature.
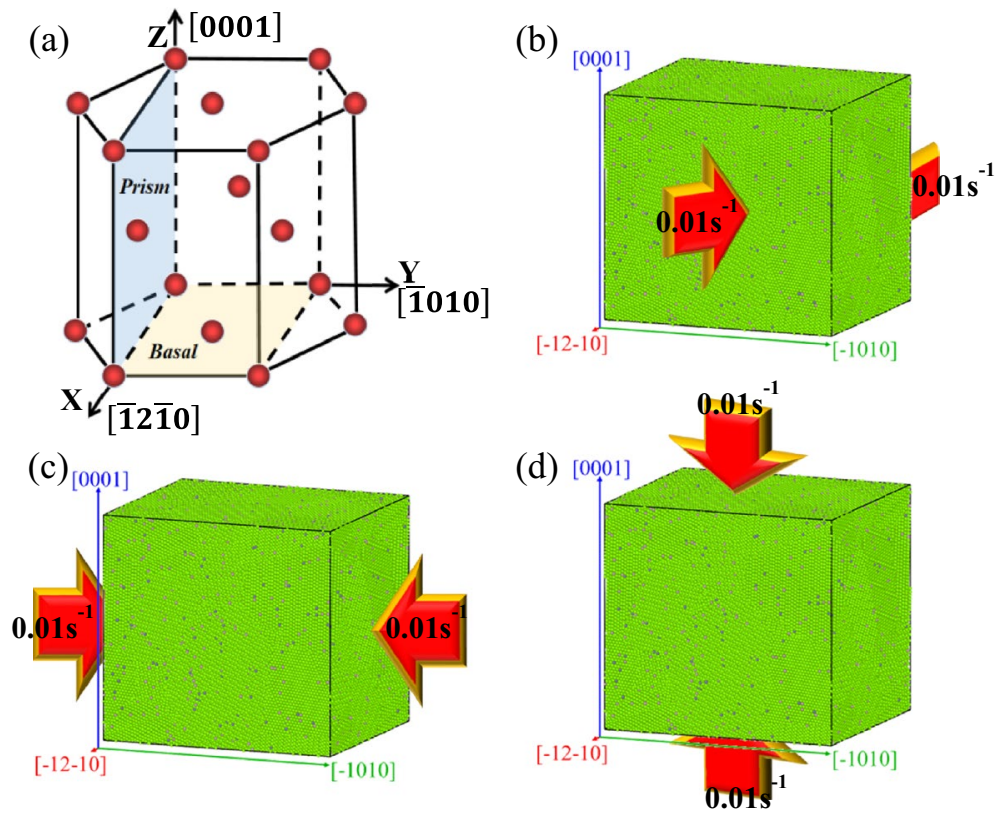

Figure 3. Diagram of the process. (a) Hexagonal closely packed cell; (b) compress along $[\overline{1} 2 \overline{1} 0] ;$ (c) compress along $[\overline{1010}]$; (d) compress along [0001].

In the formula, $E$ is the total energy of atomic $i . F$ is the embedded energy, which is a function of atomic electron density $\rho . \rho$ is atomic electron density. $\gamma$ and $\phi$ are a pair of potential interactions. And $i$ and $j$ are element types.

First of all, a Nanocrystalline Mg model with a size of $192.6 \AA \times 222.395 \AA \times 208.4 \AA$ is established ${ }^{40}$. The number of atoms is 384,020 , and the initial number of grains is 20 . The $\mathrm{X}$-axis, $\mathrm{Y}$-axis, and Z-axis are set to correspond to the crystal directions of $[\overline{1} 2 \overline{1} 0],[\overline{1} 010]$ and [0001], respectively. Then the AZ31 magnesium alloy nano-model is made by replacing 3\% $\mathrm{Mg}$ atoms with $\mathrm{Al}$ atoms and $1 \% \mathrm{Mg}$ atoms with $\mathrm{Zn}$ atoms. The established sample model is introduced into LAMMPS, and the Mg-Al-Zn potential function developed by Hyo-Sun Jang ${ }^{41}$ is assigned to the model. This potential function can be used to study the deformation and recrystallization properties of $\mathrm{Mg}-\mathrm{Al}-\mathrm{Zn}$ alloy in the whole processing temperature range. $\mathrm{X}, \mathrm{Y}$, and $\mathrm{Z}$ are all periodic boundary conditions, using the NPT ensemble. All simulations are performed using the Velocity-Verlet algorithm with a constant time step, and the ambient temperature is $300 \mathrm{~K}$. First, to create a well-equilibrated sample, the model is initially relaxed to the minimum energy configuration by the conjugate gradient method. And the model is relaxed for $10 \mathrm{ps}$ under constant temperature and pressure, The MSD curve of relaxation temperature is shown in Fig. 2. And then the strain rate of $0.01 \mathrm{ps}^{-1}$ is uniformly compressed at $20 \mathrm{ps}$ along the crystal direction [ $\left.\overline{1} 2 \overline{1} 0\right]$, $[\overline{1010}]$ and [0001] respectively. Finally, the deformation of the magnesium alloy reaches $20 \%$. The process diagram is shown in Fig. 3.

The simulation results are imported into the visualization software OVITO, and the functions: Common neighbor analysis (CNA), Dislocation analysis (DXA), Wigner-Seitz defect analysis (WSDA), and Construct surface mesh (CSM) are used for analysis. CNA provides visualization of common crystal structures in metals, 


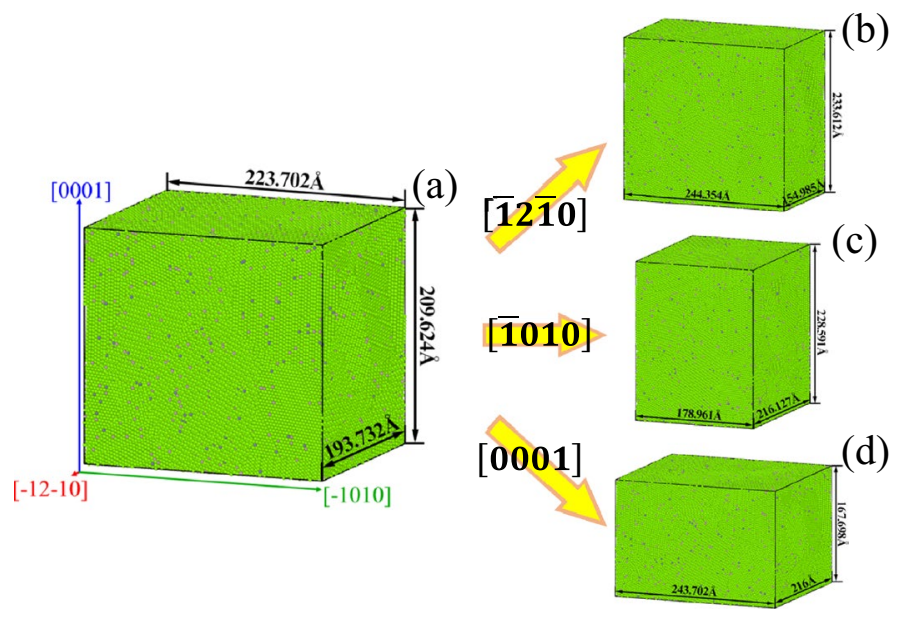

Figure 4. Compression result.

such as face-centered cubic (FCC), body-centered cubic (BCC), hexagonal closely packed (HCP) structure, and Other structure (grain boundary atoms). DXA can determine the Burgers vector of each dislocation and identify the dislocation junction. WSDA can identify the crystal gap and calculate the number of interstitial atoms. CSM can construct a three-dimensional surface formed by a group of particles to observe the voids generated during the simulation ${ }^{42-48}$.

\section{Simulation results and analysis}

Figure 4a is an undeformed AZ31 magnesium alloy with a size of $193.732 \AA \times 223.702 \AA \times 209.624 \AA$. And Fig. 4 b shows a magnesium alloy compressed $20 \%$ along $[\overline{1} 2 \overline{1} 0]$, its size becomes $154.985 \AA \times 244.354 \AA \times 233.6$ $12 \AA$. Figure $4 \mathrm{c}$ shows a magnesium alloy compressed $20 \%$ along [ 1010$]$, and its size changes to $216.127 \AA \times 17$ $8.961 \AA \times 228.591 \AA$. Figure $4 d$ shows a magnesium alloy compressed $20 \%$ along [0001], and its size changes to $216 \AA$ × $243.702 \AA \times 167.698 \AA$.

Figure 5a shows the engineering stress-engineering strain curve of AZ31 magnesium alloy under uniaxial compression in different directions, and Fig. 5b shows the Yield stress (maximum stress) and Yield strain (strain corresponding to Yield stress) of AZ31 magnesium alloy compressed in different directions. It is found that the minimum Yield stress is along $[\overline{1} 2 \overline{1} 0]$ compression, the maximum Yield stress is along $[\overline{1} 010]$ compression, but the maximum Yield strain is along [0001] compression. According to Fig. 5a, $\varepsilon<5 \%$, the elastic modulus is the same when compressed in different directions. When $5 \%<\varepsilon<U T S \%$, the elastic modulus changes ${ }^{49,50}$, and the elastic modulus is the highest when compressed along $[\overline{1} 010]$ and the lowest when compressed along $[\overline{1} 2 \overline{1} 0]$.

Figure $5 c$ shows the potential energy curve of AZ31 magnesium alloy compressed in different directions, and Fig. 5d shows the volume curve. It is found that $\varepsilon<5 \%$, When compression along with different directions, the potential energy change is the same as the volume change. And the difference between potential energy change and volume change begins to appear after $\varepsilon>5 \%$. This difference is consistent with the Ture stress-Ture strain curve. According to Fig. 5d, in the elastic stage, the volume of magnesium alloy decreases under compression, and after entering the plastic stage, the internal phase transformation of magnesium alloy occurs, forming FCC stacking fault (FCC SF), and the volume of magnesium alloy increases under compression.

Figure $6 \mathrm{a}$ is the schematic diagram of the evolution of voids in AZ31 magnesium alloy during compression in different directions. It can be found that voids are mostly formed at grain boundaries. When $\varepsilon>\mathrm{UTS} \%$, voids begin to form in magnesium alloys, and the number and area of voids increase with the increase of compression amount. Figure $6 \mathrm{~b}$ shows the voids evolution curve of AZ31 magnesium alloy during compression in different directions. It is found that the voids evolution is consistent with the change of Ture stress-Ture strain curve. $\varepsilon<5 \%$, with the increase of the amount of squeezing, the atomic position changes, and there are no voids in the model, and they are in the elastic state. $\varepsilon>5 \%$, as the squeezing continues to increase and the atomic position changes, the atoms of the HCP structure become Other structure, and the number of grain boundaries increases and voids begins to appear in the model. When $\varepsilon>\mathrm{UTS} \%$, the Other structure becomes FCC SF, the void area increases sharply, the model volume increases, and the magnesium alloy enters the plastic stage.

Figure 7 shows the CNA diagram of AZ31 magnesium alloy when it is compressed in different directions. The atoms in the picture have been colored according to CNA. The red atoms are HCP structure, the green atoms are FCC structure, and the white atoms are Other structure (that is made up the grain boundary, GB atoms). According to Fig. 7, it is found that as compression progresses, the atomic position changes, and so does the phase structure. After compression, the position of the atoms of the HCP structure changes to the atoms of the Other structure, forming the grain boundary. As the compression continues, the positions of some atoms continue to change, and the Other structure changes into the FCC structure, forming FCC SF. When $\varepsilon>\mathrm{UTS} \%$, the FCC $\mathrm{SF}$ increases rapidly. When magnesium alloy is compressed, its structure evolves to HCP $\rightarrow$ Other $\rightarrow$ FCC.

Figure 8 shows the phase structure change curve of AZ31 magnesium alloy during compression in different directions. Figure 8a shows the fractional curve of GI atoms, Fig. $8 \mathrm{~b}$ shows the fractional curve of GB atoms, 

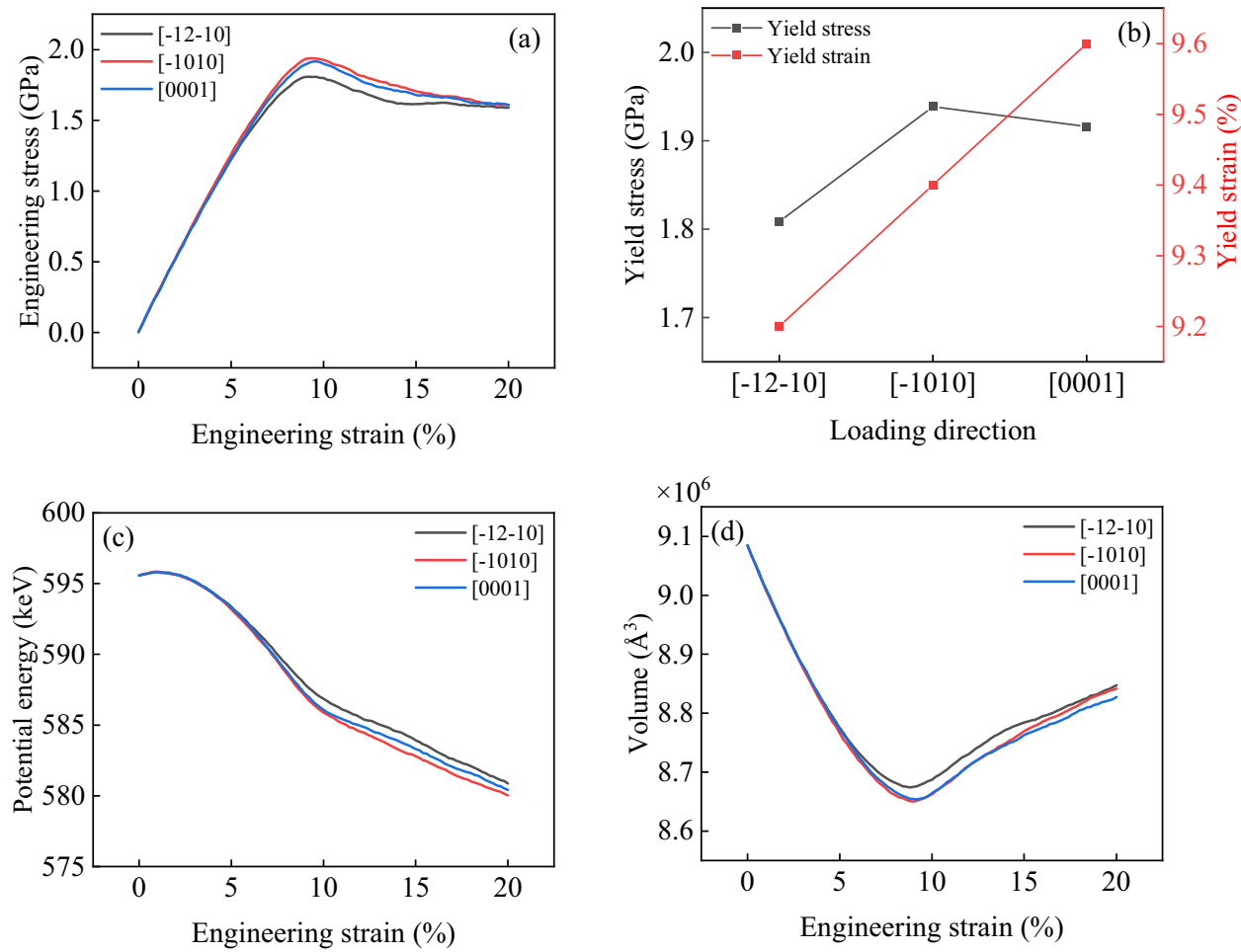

Figure 5. Performance curve. (a) Engineering stress-engineering strain curve; (b) yield stress-yield strain curve; (c) potential energy curve; (d) volume curve.

Fig. 8c-e shows the evolution of HCP structure, Fig. 8f-h shows the evolution of FCC structure, Fig. 8i shows the fractional curve of HCP structure atoms, and Fig. $8 \mathrm{j}$ shows the fractional curve of FCC structure atoms. According to Fig. $8 \mathrm{a}, \mathrm{b}$, we can see that $\varepsilon<5 \%$, with the increase of the amount of compression, the atomic fraction does not change, because there are no voids in the model and are in the elastic state. $\varepsilon>5 \%$, as the amount of compression, continues to increase, the GI atoms become GB atoms, the number of grain boundaries increases, and voids begin to appear in the model. After $\varepsilon>\mathrm{UTS} \%$, the void area increases sharply, the model volume increases, and the magnesium alloy enters the plastic state. According to Fig. $8 \mathrm{c}-\mathrm{j}$, we can see that the atoms of HCP structure decrease rapidly when $\varepsilon=5 \sim 20 \%$, while the atoms of FCC structure increase sharply when $\varepsilon=\mathrm{UTS} \% \sim 20 \%$, and the HCP transition FCC has a transition stage of about $4.5 \%$. At this stage, the HCP structure first changes into the Other structure, and when $\varepsilon>\mathrm{UTS} \%$, the Other structure becomes the FCC structure. The change of phase structure of magnesium alloy is the same when it is compressed along with all directions, and there is no obvious difference. Therefore, in the process of uniaxial compression of magnesium alloy, the phase transformation of $\mathrm{HCP} \rightarrow \mathrm{FCC}$ is the main one.

Figure 9 shows the dislocation evolution and density curve of AZ31 magnesium alloy during compression along $[\overline{1} 2 \overline{1} 0]$, Fig. 9a shows the dislocation evolution in the model, the atoms in the figure have been treated transparently, and Fig. $9 \mathrm{~b}$ shows the dislocation density curve. According to Fig. 9a, it can be found that the most obvious stage of dislocation growth is $\varepsilon=5 \sim 20 \%$. As the number of compression increases, the atomic position changes, and the number and length of dislocations increase. According to Fig. $9 \mathrm{~b}$, it can be found that $\varepsilon<5 \%$, with the increase of compression, the dislocations in the model are mainly Other dislocations, that is unrecognizable dislocations. At this time, what happens in the model is the transition of HCP $\rightarrow$ Other, which is in an elastic state. $\varepsilon>5 \%$, with the increase of compression amount, there are $1 / 3<\overline{1} 100>$ dislocations(Shockley partial dislocations) in the model, Other structure increases, and the number of grain boundaries increases. When $\varepsilon>\mathrm{UTS} \%$, part of the Other structure becomes FCC SF, and the magnesium alloy enters the plastic state. According to Fig. 8, the dislocation evolution of magnesium alloy during compression in all directions is consistent, and there is no significant difference in dislocation density.

Figure 10a shows the growth of Shockley partial dislocations and FCC SF of AZ31 magnesium alloy during compression along $[\overline{1} 2 \overline{1} 0]$. To observe the positions of dislocations and SF, the atoms are treated transparently, and the positions of Shockley partial dislocations and FCC SF are marked. It can be found that Shockley partial dislocations always surround FCC SF. With the increase of the amount of compression, the atoms of HCP structure first become Other structure, resulting in Shockley partial dislocations, the atomic position changes continuously, the dislocations move, and the atoms of Other structure become FCC structure, forming FCC SF.

Figure 10b shows the curves of the growth of Shockley partial dislocations and FCC SF under different compression directions. It is found that Shockley partial dislocations always occur about $4.5 \%$ earlier than FCC SF. This is because the atomic position changes during compression, but does not directly change from HCP phase structure to FCC phase structure, but slips first to become Other structure atoms, resulting in Shockley partial 
(a)

[12̄10]

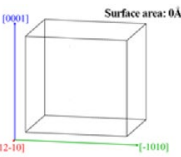

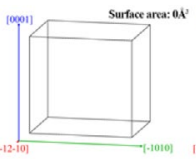
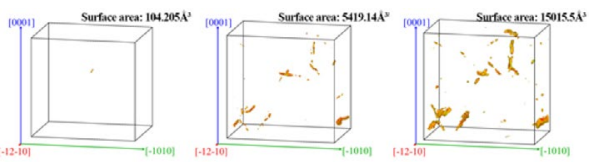

[1010]
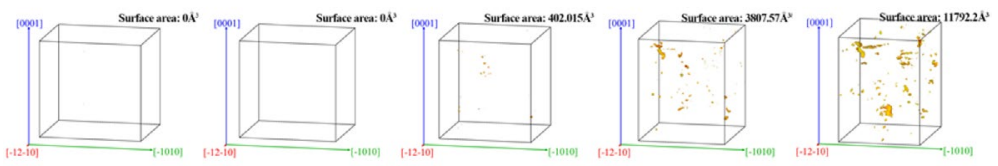

[0001]
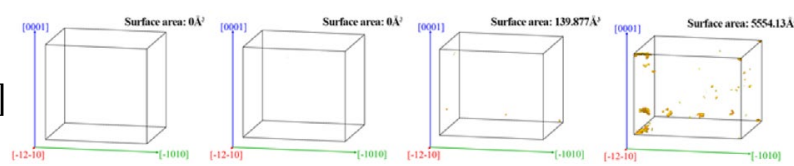

$\varepsilon=0 \%$

$\varepsilon=\mathrm{UTS} \%$

$\varepsilon=15 \%$

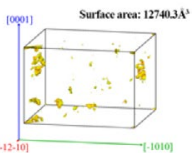

(b)

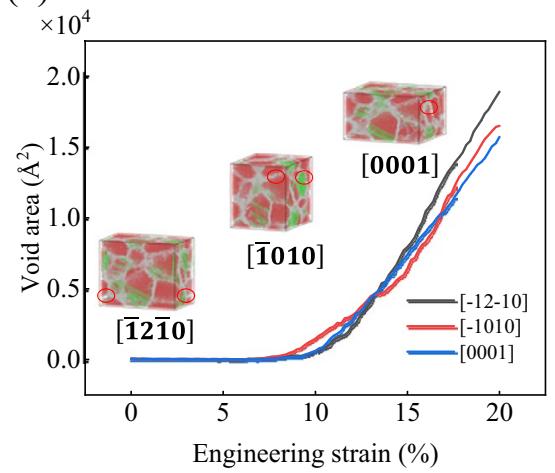

Figure 6. Evolution of voids.

(a)

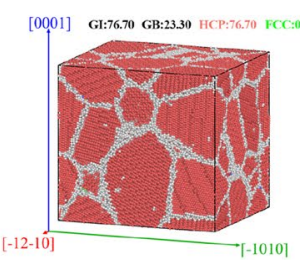

(b)

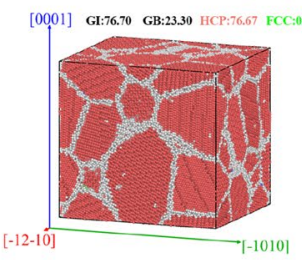

(c)
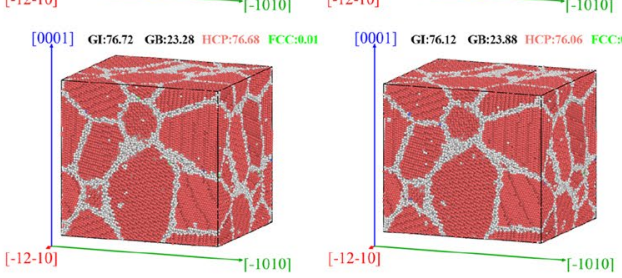

$\varepsilon=0 \%$
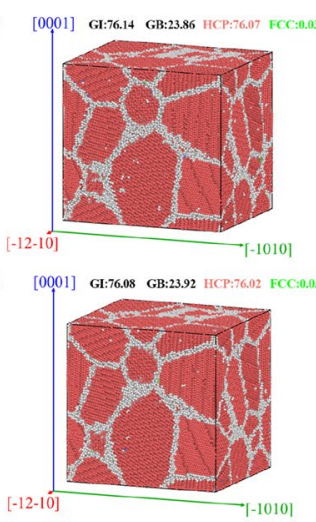

$\varepsilon=5 \%$
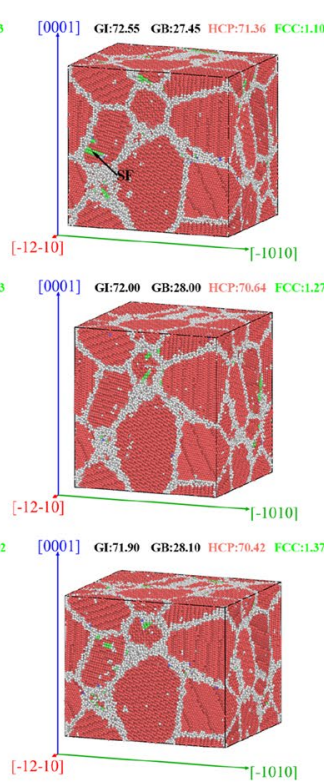

$\varepsilon=\mathrm{UTS} \%$
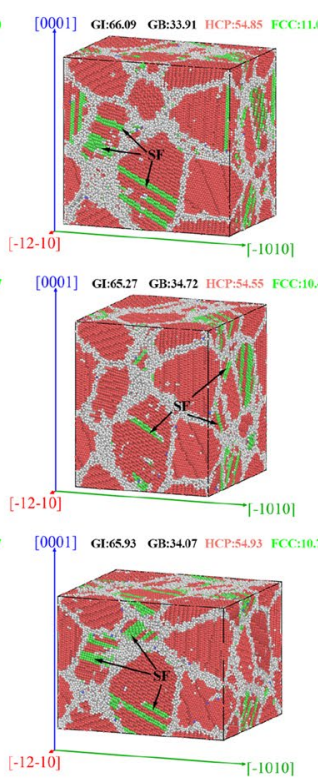

$\varepsilon=15 \%$
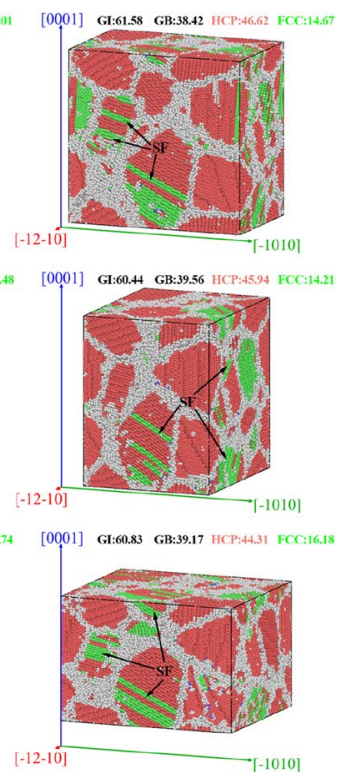

$\varepsilon=20 \%$

Figure 7. CNA diagram of AZ31 magnesium alloy during compression. (a) Compress along [ $\overline{1} 2 \overline{1} 0]$; (b) compress along $[\overline{1} 010] ;$ (c) compress along [0001].

dislocations. With the increase of compression, Shockley partial dislocations increase. When $\varepsilon>\mathrm{UTS} \%$, the FCC SF is formed and the volume increases.

\section{Conclusion}

In this paper, to study the effect of loading direction on uniaxial compression of AZ31 magnesium alloy, the model of AZ31 magnesium alloy was established. The uniaxial compression process of magnesium alloy along $[\overline{1} 2 \overline{1} 0],[\overline{1} 010]$ and $[0001]$ was simulated by molecular dynamics, the changes of atomic position and phase structure were observed, and the phase transformation mechanism of AZ31 magnesium alloy under different loading directions was summarized. The main results are as follows:

(1) Engineering stress-engineering strain, potential energy and volume change, void evolution, phase structure change, and dislocation evolution of magnesium alloy are consistent with each direction of compression, and there is no obvious difference.

(2) $\varepsilon<5 \%$, the atomic position changes with the increase of compression, the dislocations in the model are mainly Other dislocations, that is unrecognizable dislocations, what happens at this time is the transition of HCP structure $\rightarrow$ Other structure. There are no voids in the model and are in an elastic state. $\varepsilon>5 \%$ The atom of the HCP structure becomes Other structure. $1 / 3<\overline{1} 100>$ dislocations are generated in the model (Shockley partial dislocations). And the number of grain boundaries increases, and the voids begin 


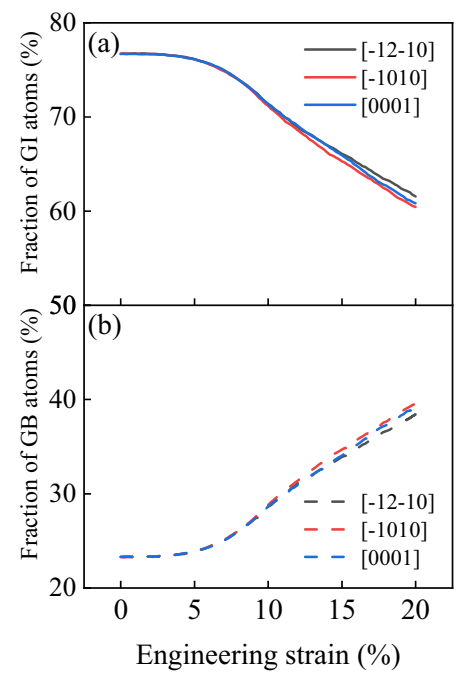

(c)

(d)

(e)

(f)

(g)

(h)
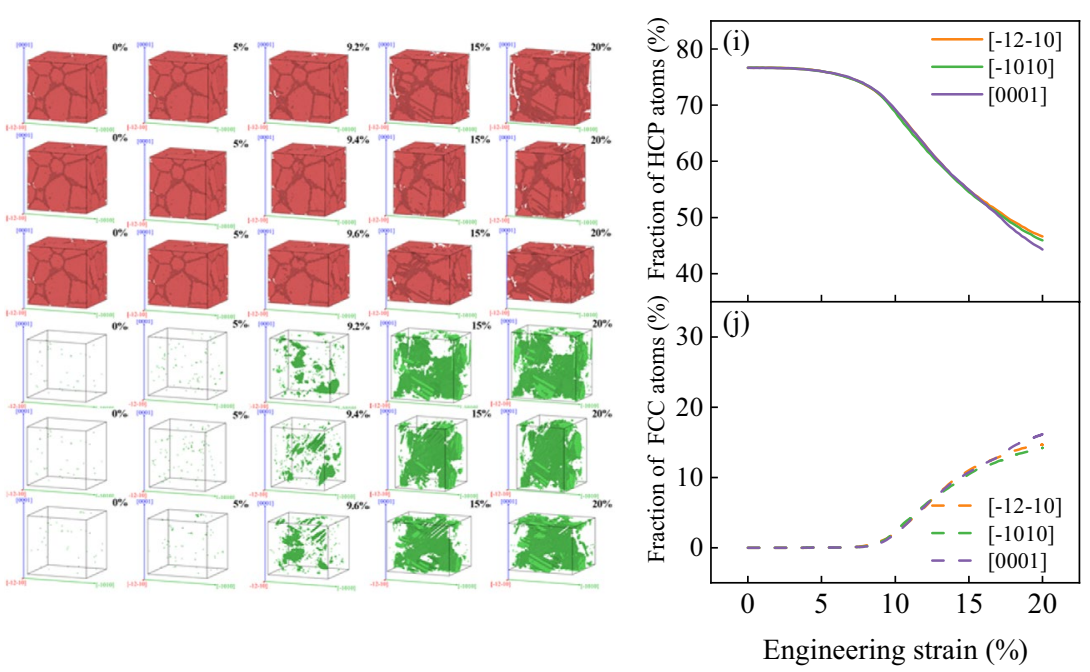

Figure 8. Phase structure change curve during compression. (a) Fractional curve of GI atoms; (b) fractional curve of GB atoms; (c) (e) HCP structure evolution; (f) (h) FCC structure evolution; (i) fractional curve of HCP structure atoms; (j) fractional curve of FCC structure atoms.

(a)
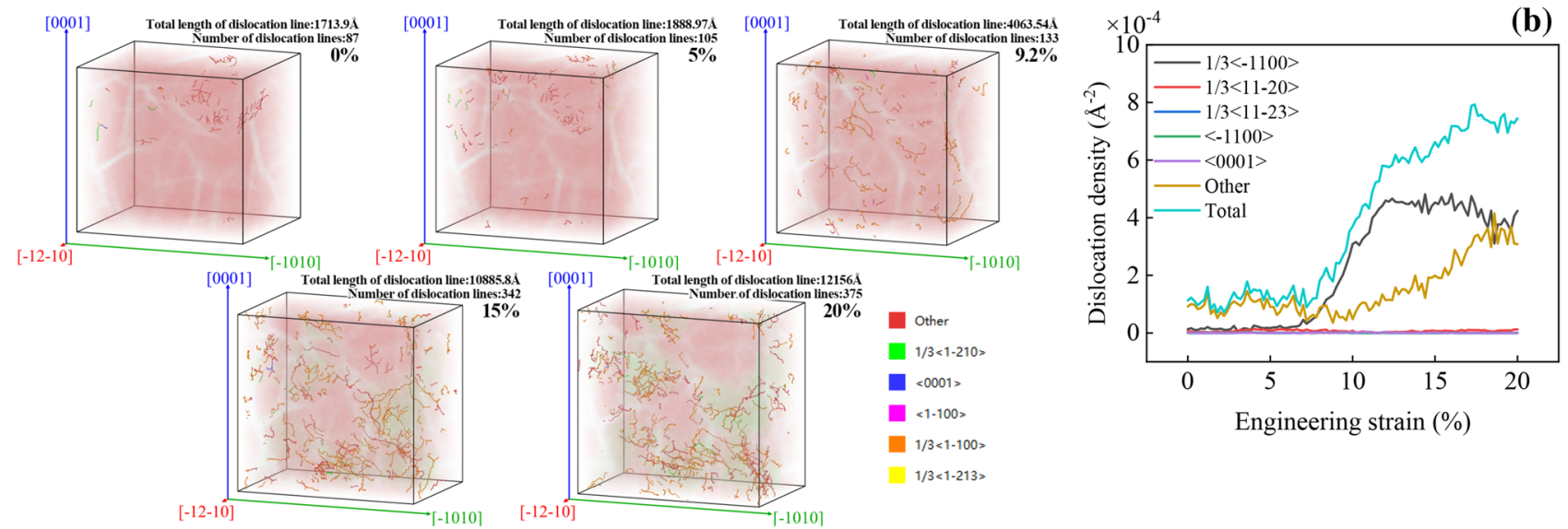

Figure 9. Dislocation evolution and density curve during compression along [ $\overline{1} 2 \overline{1} 0]$.

(a)

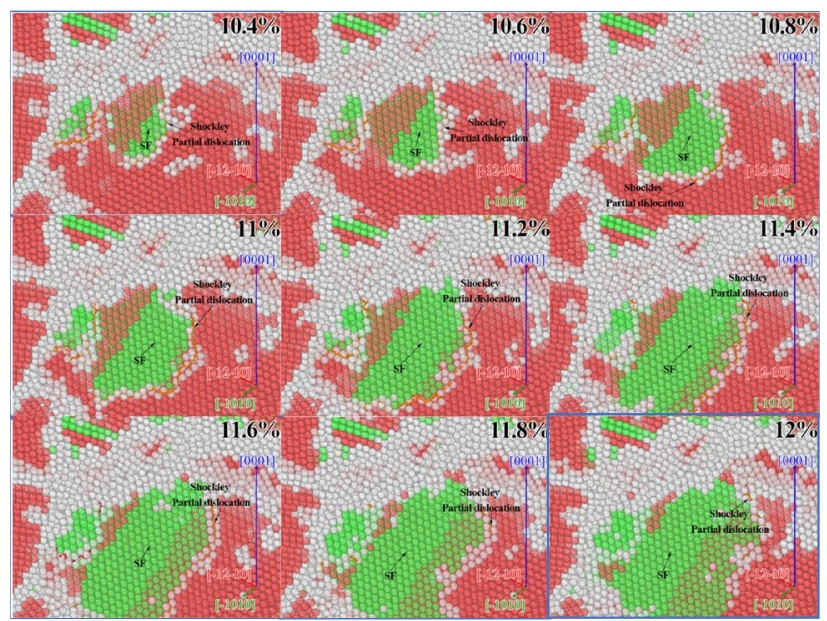

(b)

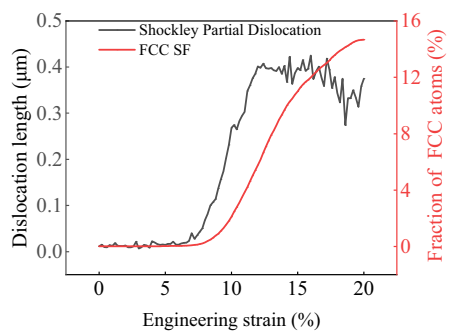

Figure 10. Correlation between Shockley partial dislocations and FCC SF. 
to appear at this time. $\varepsilon>$ UTS\%, the Other structure becomes FCC SF. the void area increases sharply, the model volume increases, and the magnesium alloy enters the plastic state.

(3) In the process of uniaxial compression of magnesium alloy, the phase transformation of $\mathrm{HCP} \rightarrow \mathrm{FCC}$ is the main. $\mathrm{HCP} \rightarrow \mathrm{FCC}$ has a transition stage of about $4.5 \%$, and its structure evolves into $\mathrm{HCP} \rightarrow$ Other $\rightarrow$ FCC. The atoms of the HCP structure are compressed and become the atoms of the Other structure, forming the grain boundary. With the continuation of the compression, the Other structure becomes the FCC structure, forming the FCC SF. When $\varepsilon>\mathrm{UTS} \%$, the FCC SF increases rapidly.

(4) Shockley partial dislocations always occur about $4.5 \%$ before FCC SF, and Shockley partial dislocations surround FCC SF.

\section{Data availability}

The datasets generated during and/or analysed during the current study are available from the corresponding author on reasonable request.

Received: 28 May 2021; Accepted: 11 August 2021

Published online: 26 August 2021

\section{References}

1. Nirala, A. et al. Comprehensive study of magnesium based metal matrix composite. Mater. Today Proc. 46(15), 6587-6591 (2021).

2. Tiancai, Xu., Yang, Y., Peng, X., Song, J. \& Pan, F. Overview of advancement and development trend on magnesium alloy. J. Magnes. Alloys. 7(3), 536-544 (2019).

3. Sankaranarayanan, S. \& Gupta, M. Emergence of god's favorite metallic element: Magnesium based materials for engineering and biomedical applications. Mater. Today Proc. 39, 311-316 (2021).

4. Tekumalla, S. \& Gupta, M. Processing, properties and potential applications of magnesium alloy-based nanocomposites: A review. In Nanocomposites VI: Nanoscience andnanotechnology in advanced composites (eds. Srivatsan, T. \& Gupta, M.) (The Minerals, Metals \& Materials Series. Springer, Cham. 2019)

5. Luo, Q. et al. Thermodynamics and kinetics of phase transformation in rare earth-magnesium alloys: A critical review. J. Mater. Sci. Technol. 44, 171-190 (2020).

6. Ravichandrana, N. \& Mungara, S. R. Manufacturing and deployment of magnesium alloy parts for light weighting applications. Mater. Today Proc. (2021).

7. Kumar, D., Phanden, R. K. \& Thakur, L. A review on environment friendly and lightweight magnesium-based metal matrix composites and alloys. Mater. Today Proc. 38, 359-364 (2021).

8. Yang, Y. et al. Research advances in magnesium and magnesium alloys worldwide in 2020. J. Magnes. Alloys. 9(3), 705-747 (2021).

9. Cerreta, E. K. et al. The high-strain-rate constitutive behavior and shear response of pure magnesium and AZ31B magnesium alloy. Metall. Mater. Trans. A. 52(7), 3152-3170 (2021).

10. Gleiter, H. Nanostructured materials: Basic concepts and microstructure. Acta Mater. 48(1), 1-29 (2000).

11. Wenwu, Xu., Song, X., Nianduan, Lu. \& Huang, C. Thermodynamic and experimental study on phase stability in nanocrystalline alloys. Acta Mater. 58(2), 396-407 (2010).

12. Song, X. et al. Effect of interfacial characteristics on toughness of nanocrystalline cemented carbides. Acta Mater. 61(6), 2154-2162 (2013).

13. Suryanarayana, C. Structure and properties of nanocrystalline materials. Bull. Mater. Sci. 17, 307-346 (1994).

14. Dong, Y. \& Chen, I. W. Onset criterion for flash sintering. J. Am. Ceram. Soc. 98, 3624-3627 (2018).

15. Mahata, A. \& Sikdar, K. Molecular dynamics simulation of nanometer-scale mechanical properties of hexagonal Mg-Li alloy. J. Magnes. Alloys. 4(1), 36-43 (2016).

16. Mansoor, P. \& Dasharath, S. M. A review paper on magnesium alloy fabricated by severe plastic deformation technology and its effects over microstructural and mechanical properties. Mater. Today Proc. 45, 356-364 (2021).

17. Susumu, K. Molecular mechanical-engineering. JSME Int J. Ser. B 38(1), 1-7 (1995).

18. Wolf, D., Yamakov, V., Phillpot, S. R., Mukherjee, A. \& Gleiter, H. Deformation of nanocrystalline materials by molecular-dynamics simulation: Relationship to experiments?. Acta Mater. 53(1), 1-40 (2005).

19. Silvestre, N., Faria, B. \& Canongia Lopes, J. N. Compressive behavior of CNT-reinforced aluminum composites using molecular dynamics. Compos. Sci. Technol. 90, 16-24 (2014).

20. Yang, X. et al. Molecular dynamics modeling of mechanical and tribological properties of additively manufactured AlCoCrFe high entropy alloy coating on aluminum substrate. Mater. Chem. Phys. 263, 124341 (2021).

21. Lucchetta, A., Brach, S. \& Kondo, D. Effects of particles size on the overall strength of nanocomposites: Molecular dynamics simulations and theoretical modeling. Mech. Res. Commun. 114, 103669 (2021).

22. Yamakov, V., Wolf, D., Phillpot, S. R., Mukherjee, A. K. \& Gleiter, H. Deformation-mechanism map for nanocrystalline metals by molecular-dynamics simulation. Nat. Mater. 3, 43-47 (2004).

23. Yamakov, V., Wolf, D., Phillpot, S. R., Mukherjee, A. K. \& Gleiter, H. Dislocation processes in the deformation of nanocrystalline aluminium by molecular-dynamics simulation. Nat. Mater. 1, 45-49 (2002).

24. Zheng, G. P., Wang, Y. M. \& Li, M. Atomistic simulation studies on deformation mechanism of nanocrystalline cobalt. Acta Mater. 53(14), 3893-3901 (2005).

25. Rawat, S. \& Chaturvedi, S. Strain-rate effect on plasticity and $\omega$-phase transformation in single crystal titanium: A molecular dynamics study. Mech. Mater. 148, 103529 (2020).

26. Xiaoqin, Ou. \& Song, M. Deformation mechanisms of mechanically induced phase transformations in iron. Comput. Mater. Sci. 162, 12-20 (2019).

27. Mojumder, S. Molecular dynamics study of plasticity in Al-Cu alloy nanopillar due to compressive loading. arXiv:1707.08404 [cond-mat.mtrl-sci].

28. Zhang, H., Ou, X., Wei, B., Ni, S. \& Song, M. Strain direction dependency of deformation mechanisms in an HCP-Ti crystalline by molecular dynamics simulations. Comput. Mater. Sci. 172, 109328 (2020).

29. Plimpton, S. Fast parallel algorithms for short-range molecular dynamics. J. Comput. Phys. 117(1), 1-19 (1995).

30. Caro, A., Crowson, D. A. \& Caro, M. Classical many-body potential for concentrated alloys and the inversion of order in ironchromium alloys. Phys. Rev. Lett. 95, 075702 (2005).

31. Stukowski, A., Sadigh, B., Erhart, P. \& Caro, A. Efficient implementation of the concentration-dependent embedded atom method for molecular-dynamics and Monte-Carlo simulations. Model. Simul. Mater. Sci. Eng. 17, 075005 (2009).

32. Zhou, X. W., Johnson, R. A. \& Wadley, H. N. G. Misfit-energy-increasing dislocations in vapor-deposited CoFe/NiFe multilayers. Phys. Rev. B. 69, 144113 (2004). 
33. Baskes, M. I. Modified embedded-atom potentials for cubic materials and impurities. Phys. Rev. B 46, 2727 (1992).

34. Fang, H. et al. Numerical tools for atomistic simulations. United States:N (2004).

35. Lee, B.-J. \& Baskes, M. I. Second nearest-neighbor modified embedded-atom-method potential. Phys. Rev. B. 62, 8564 (2000).

36. Lee, B.-J., Baskes, M. I., Kim, H. \& Cho, Y. K. Second nearest-neighbor modified embedded atom method potentials for bcc transition metals. Phys. Rev. B. 64, 184102 (2001).

37. Valone, S. M., Baskes, M. I. \& Martin, R. L. Atomistic model of helium bubbles in gallium-stabilized plutonium alloys. Phys. Rev. B. 76, 214209 (2006).

38. Wang, G., Van Hove, M. A., Ross, P. N. \& Baskes, M. I. Monte carlo simulations of segregation in Pt-Re catalyst nanoparticles. J. Chem. Phys. 121, 5410 (2004).

39. Ziegler, J. F. \& Biersack, J. P. The stopping and range of ions in matter. In Treatise on Heavy-Ion Science (ed. Bromley, D. A.) 93-129 (Springer, 1985).

40. Hirel, P. Atomsk: A tool for manipulating and converting atomic data files. Comput. Phys. Commun. 197, 212-219 (2015).

41. Jang, H.-S., Seol, D. \& Lee, B.-J. Modified embedded-atom method interatomic potentials for Mg-Al-Ca and Mg-Al-Zn ternary systems. J. Magnes. Alloys. 9(1), 317-335 (2021).

42. Stukowski, A. Visualization and analysis of atomistic simulation data with OVITO-the Open Visualization Tool. Model. Simul. Mater. Sci. Eng. 18(1), 015012 (2009).

43. Larsen, P. M., Schmidt, S. \& Schiøtz, J. Robust structural identification via polyhedral template matching. Model. Simul. Mater. Sci. Eng. 24(5), 055007 (2016).

44. Bonald, T., Charpentier, B., Galland, A. \& Hollocou, A. Hierarchical graph clustering using node pair sampling. arXiv:1806.01664 [cs.SI].

45. Panzarino, J. F. \& Rupert, T. J. Tracking microstructure of crystalline materials: A post-processing algorithm for atomistic simulations. JOM. 66, 417-428 (2014).

46. Stukowski, A., Bulatov, V. V. \& Arsenlis, A. Automated identification and indexing of dislocations in crystal interfaces. Model. Simul. Mater. Sci. Eng. 20(8), 085007 (2012).

47. Honeycutt, J. D. \& Andersen, H. C. Molecular dynamics study of melting and freezing of small Lennard-Jones clusters. J. Phys. Chem. 91(19), 4950-4963 (1987).

48. Stukowski, A. Computational analysis methods in atomistic modeling of crystals. JOM. 66(3), 399-407 (2014).

49. Sun, X., Xu, G., Li, X., Feng, X. \& Gao, H. Mechanical properties and scaling laws of nanoporous gold. J. Appl. Phys. 113, 023505 (2013).

50. Lia, J. et al. Structurally ordered nanoporous Pt-Co alloys with enhanced mechanical behaviors in tension. Microporous Mesoporous Mater. 295, 109955 (2020).

\section{Acknowledgements}

This work was supported by the National Key Research and Development Project (2018YFB1307902), Shanxi Province Joint Student Training Base Talent Training Project (No. 2018JD33), Shanxi young top talent project, Shanxi Province Science Foundation for Youths (201901D211312), Excellent young academic leaders in Shanxi colleges and universities (No. 2019045), Excellent Achievements Cultivation Project of Shanxi Higher Education Institutions (No. 2019KJ028), Shanxi Province emerging industry leader talent project, Shanxi Graduate Education Innovation Project (No. 2019SY482).

\section{Author contributions}

Q.H.Y. ideated, designed the study and analyzed the results. Q.H.Y. carried out the MC/MD structure evolution analysis, dislocation visualization analysis and making the figures. Z.B.C. contributed to the overall guidance for the work and provided financial support. C.X., Y.G.L., L.F.M. and H.G. provided ideas.

\section{Competing interests}

The authors declare no competing interests.

\section{Additional information}

Correspondence and requests for materials should be addressed to Z.C.

Reprints and permissions information is available at www.nature.com/reprints.

Publisher's note Springer Nature remains neutral with regard to jurisdictional claims in published maps and institutional affiliations.

(1) Open Access This article is licensed under a Creative Commons Attribution 4.0 International cc) License, which permits use, sharing, adaptation, distribution and reproduction in any medium or format, as long as you give appropriate credit to the original author(s) and the source, provide a link to the Creative Commons licence, and indicate if changes were made. The images or other third party material in this article are included in the article's Creative Commons licence, unless indicated otherwise in a credit line to the material. If material is not included in the article's Creative Commons licence and your intended use is not permitted by statutory regulation or exceeds the permitted use, you will need to obtain permission directly from the copyright holder. To view a copy of this licence, visit http://creativecommons.org/licenses/by/4.0/.

(c) The Author(s) 2021 